\title{
A SUBCLASS OF HARMONIC FUNCTIONS WITH NEGATIVE COEFFICIENTS DEFINED BY DZIOK-SRIVASTAVA OPERATOR
}

\author{
G. MURUGUSUNDARAMOORTHY, K. VIJAYA AND B. A. FRASIN
}

\begin{abstract}
Making use of the Dziok-Srivastava operator, we introduce the class $\mathscr{R} \frac{p, q}{\not \mathscr{H}}$ ([ $\left.\left.\alpha_{1}\right], \lambda, \gamma\right)$ of complex valued harmonic functions. We investigate the coefficient bounds, distortion inequalities, extreme points and inclusion results for this class.
\end{abstract}

\section{Introduction}

A continuous function $f=u+i v$ is a complex- valued harmonic function in a complex domain $\Omega$ if both $u$ and $v$ are real and harmonic in $\Omega$. In any simply-connected domain $D \subset \Omega$, we can write $f=h+\bar{g}$, where $h$ and $g$ are analytic in $D$. We call $h$ the analytic part and $g$ the co-analytic part of $f$. A necessary and sufficient condition for $f$ to be locally univalent and orientation preserving in $D$ is that $\left|h^{\prime}(z)\right|>\left|g^{\prime}(z)\right|$ in $D$ (see [3]).

Denote by $\mathscr{H}$ the family of functions

$$
f=h+\bar{g}
$$

which are harmonic, univalent and orientation preserving in the open unit disc $U=\{z:|z|<$ $1\}$ so that $f$ is normalized by $f(0)=h(0)=f_{z}(0)-1=0$. Thus, for $f=h+\bar{g} \in \mathscr{H}$, we may express the analytic functions for $h$ and $g$ in the forms

$$
h(z)=z+\sum_{n=2}^{\infty} a_{n} z^{n}, g(z)=\sum_{n=1}^{\infty} b_{n} z^{n} \quad\left(\left|b_{1}\right|<1\right) .
$$

Hence

$$
f(z)=z+\sum_{n=2}^{\infty} a_{n} z^{n}+\overline{\sum_{n=1}^{\infty} b_{n} z^{n}},\left|b_{1}\right|<1 .
$$

We note that the family $\mathscr{H}$ of orientation preserving, normalized harmonic univalent functions reduces to the well known class $S$ of normalized univalent functions if the co-analytic part of $f$ is identically zero, that is $g \equiv 0$.

Corresponding author: G. Murugusundaramoorthy. 2010 Mathematics Subject Classification. Primary 30C45, 30C50.

Key words and phrases. Harmonic univalent starlike functions, Dziok-Srivastava operator, distortion bounds, extreme points, convolution. 
Also denote by $\overline{\mathscr{H}}$ the subfamily of $\mathscr{H}$ consists harmonic functions $f=h+\bar{g}$ of the form

$$
f(z)=z-\sum_{n=2}^{\infty}\left|a_{n}\right| z^{n}+\overline{\sum_{n=1}^{\infty}\left|b_{n}\right| z^{n}},\left|b_{1}\right|<1 .
$$

Let the Hadamard product (or convolution) of two power series

$$
\phi(z)=z+\sum_{n=2}^{\infty} \phi_{n} z^{n}
$$

and

$$
\psi(z)=z+\sum_{n=2}^{\infty} \psi_{n} z^{n}
$$

be defined by

$$
(\phi * \psi)(z)=\phi(z) * \psi(z)=z+\sum_{n=2}^{\infty} \phi_{n} \psi_{n} z^{n}
$$

For complex parameters $\alpha_{1}, \ldots, \alpha_{p}$ and $\beta_{1}, \ldots, \beta_{q}\left(\beta_{j} \neq 0,-1, \ldots ; j=1,2, \ldots, q\right)$ the generalized hypergeometric function ${ }_{p} F_{q}(z)$ is defined by

$$
\begin{gathered}
{ }_{p} F_{q}(z) \equiv{ }_{p} F_{q}\left(\alpha_{1}, \ldots, \alpha_{p} ; \beta_{1}, \ldots, \beta_{q} ; z\right):=\sum_{n=0}^{\infty} \frac{\left(\alpha_{1}\right)_{n} \ldots\left(\alpha_{p}\right)_{n}}{\left(\beta_{1}\right)_{n} \ldots\left(\beta_{q}\right)_{n}} \frac{z^{n}}{n !}, \\
\left(p \leq q+1 ; p, q \in N_{0}:=N \cup\{0\} ; z \in U\right)
\end{gathered}
$$

where $N$ denotes the set of all positive integers and $(a)_{n}$ is the Pochhammer symbol defined by

$$
(a)_{n}= \begin{cases}1, & n=0 \\ a(a+1)(a+2) \ldots(a+n-1), & n \in N .\end{cases}
$$

Let

$$
H\left(\alpha_{1}, \ldots, \alpha_{p} ; \beta_{1}, \ldots, \beta_{q}\right): A \rightarrow A
$$

be a linear operator defined by

$$
\begin{aligned}
{\left[\left(H\left(\alpha_{1}, \ldots, \alpha_{p} ; \beta_{1}, \ldots, \beta_{q}\right)\right)(\phi)\right](z):=} & z_{p} F_{q}\left(\alpha_{1}, \alpha_{2}, \ldots, \alpha_{p} ; \beta_{1}, \beta_{2}, \ldots, \beta_{q} ; z\right) * \phi(z) \\
= & z+\sum_{n=2}^{\infty} \Gamma\left(\alpha_{1}, n\right) a_{n} z^{n}
\end{aligned}
$$

where

$$
\Gamma\left(\alpha_{1}, n\right)=\frac{\left(\alpha_{1}\right)_{n-1} \ldots\left(\alpha_{p}\right)_{n-1}}{\left(\beta_{1}\right)_{n-1} \ldots\left(\beta_{q}\right)_{n-1}} \frac{1}{(n-1) !}
$$

and

$$
\alpha_{i}>0(i=1,2, \ldots p), \beta_{j}>0(j=1,2, \ldots q), p \leq q+1 ; p, q \in N_{0}=N \cup\{0\} .
$$


For notational simplicity, we use a shorter notation $H_{q}^{p}\left[\alpha_{1}\right]$ for $H\left(\alpha_{1}, \ldots, \alpha_{p} ; \beta_{1}, \ldots, \beta_{q}\right)$ in the sequel. Note that if $q=0$ then

$$
H_{0}^{p}\left[\alpha_{1}\right]=z+\sum_{n=2}^{\infty} \frac{\left(\alpha_{1}\right)_{n-1} \ldots\left(\alpha_{p}\right)_{n-1}}{(n-1) !} a_{n} z^{n}
$$

It follows from (1.6) that

$$
H_{0}^{1}[1] f(z)=f(z), H_{0}^{1}[2] f(z)=z f^{\prime}(z) \text { and } H_{0}^{1}[3] f(z)=z f^{\prime}(z)+\frac{1}{2} z^{2} f^{\prime \prime}(z) .
$$

The linear operator $H_{q}^{p}\left[\alpha_{1}\right]$ is the Dziok-Srivastava operator (see [4]), which contains such well known operators as the Hohlov linear operator, Saitoh's generalized linear operator, the Carlson-Shaffer linear operator, the Ruscheweyh derivative operator as well as its generalized versions, the Bernardi-Libera-Livingston operator, and the Srivastave-Owa fractional derivative operator. One may refer to Carlson and Shaffer [2], Dziok and Srivastava[4] and Srivastava and Owa [15] for more details concerning these operators (see also [1, 11, 12, 13]).

Applying the Dziok-Srivastava operator to the harmonic function $f=h+\bar{g}$ given by (1.1), we readily get

$$
H_{q}^{p}\left[\alpha_{1}\right] f(z)=H_{q}^{p}\left[\alpha_{1}\right] h(z)+\overline{H_{q}^{p}\left[\alpha_{1}\right] g(z)}
$$

Motivated by the earlier works of $[5,6,7,8,14,16]$ on the subject of harmonic functions, we introduce here a new subclass $\mathscr{R}_{\mathscr{H}}^{p, q}\left(\left[\alpha_{1}\right], \lambda, \gamma\right)$ of $\mathscr{H}$.

For $0 \leq \gamma<1$, we let $\mathscr{R}_{\mathscr{H}}^{p, q}\left(\left[\alpha_{1}\right], \lambda, \gamma\right)$ a subclass of $\mathscr{H}$ of the form $f=h+\bar{g}$ given by (1.2) and satisfying the analytic criteria

$$
\operatorname{Re}\left\{\frac{z\left(H_{q}^{p}\left[\alpha_{1}\right] h(z)\right)^{\prime}-z \overline{\left(H_{q}^{p}\left[\alpha_{1}\right] g(z)\right)^{\prime}}}{(1-\lambda)\left(H_{q}^{p}\left[\alpha_{1}\right] h(z)+\overline{H_{q}^{p}\left[\alpha_{1}\right] g(z)}\right)+\lambda\left(z\left(H_{q}^{p}\left[\alpha_{1}\right] h(z)\right)^{\prime}-\overline{z\left(H_{q}^{p}\left[\alpha_{1}\right] g(z)\right)^{\prime}}\right)}\right\} \geq \gamma
$$

where $0 \leq \lambda \leq 1, H_{q}^{p}\left[\alpha_{1}\right] f(z)$ is given by (1.2) and $z \in U$.

We also let $\mathscr{R}_{\mathscr{\not}}^{p, q}\left(\left[\alpha_{1}\right], \lambda, \gamma\right)=\mathscr{R}_{\mathscr{H}}^{p, q}\left(\left[\alpha_{1}\right], \lambda, \gamma\right) \cap \overline{\mathscr{H}}$.

The family $\mathscr{R} \frac{p, q}{\mathscr{H}}\left(\left[\alpha_{1}\right], \lambda, \gamma\right)$ is of special interest because it reduces to various classes of well-known harmonic univalent functions as well many new ones. For example:

(i) $\mathscr{R} \frac{1,0}{\mathscr{H}}\left(\left[\alpha_{1}\right], 0, \gamma\right) \equiv \mathcal{V} \overline{\mathscr{H}}_{(}\left(\alpha_{1}, \gamma\right) \quad$ (Al-Kharsani and Al-Khal[9]), item $\mathscr{R} \frac{1,0}{\mathscr{H}}([1], \lambda, \gamma) \equiv \mathscr{T}_{\mathscr{S}^{*}}^{*}(\lambda, \gamma)$ (Öztürk et al.[10]),

(ii) $\mathscr{R}_{\mathscr{H}}^{1,0}([1], 0, \gamma) \equiv \mathscr{T}_{\mathscr{H}}(\gamma) \quad$ (Jahangiri [6]),

(iii) $\mathscr{R} \frac{1,0}{\mathscr{H}}([1], 0,0) \equiv \mathscr{T}_{\mathscr{H}}^{* 0} \quad$ (Silverman [14]). 
In this paper, we obtained coefficient conditions for the classes $\mathscr{R}_{\mathscr{H}}^{p, q}\left(\left[\alpha_{1}\right], \lambda, \gamma\right)$ and $\mathscr{R} \frac{p, q}{\mathscr{H}}\left(\left[\alpha_{1}\right], \lambda, \gamma\right)$. Coefficient bounds, distortion inequalities, inclusion properties for the class $\mathscr{R} \frac{p, q}{\mathscr{H}}\left(\left[\alpha_{1}\right], \lambda, \gamma\right)$ are also established.

\section{Coefficient bounds}

In our first theorem, we obtain a sufficient coefficient condition for harmonic functions in $\mathscr{R}_{\mathscr{H}}^{p, q}\left(\left[\alpha_{1}\right], \lambda, \gamma\right)$.

Theorem 2.1. Let $f=h+\bar{g}$ be given by (1.2). If

$$
\sum_{n=1}^{\infty}\left[(n-\gamma-\gamma \lambda(n-1))\left|a_{n}\right|+(n+\gamma-\gamma \lambda(n+1))\left|b_{n}\right|\right] \Gamma\left(\alpha_{1}, n\right) \leq 2(1-\gamma)
$$

where $a_{1}=1$ and $0 \leq \gamma<1$, then $f \in \mathscr{R}_{\mathscr{H}}^{p, q}\left(\left[\alpha_{1}\right], \lambda, \gamma\right)$.

Proof. We first show that if (2.1) holds for the coefficients of $f=h+\bar{g}$, the required condition (1.8) is satisfied. From (1.8) we can write

$$
\begin{aligned}
& \operatorname{Re}\left\{\frac{z\left(H_{q}^{p}\left[\alpha_{1}\right] h(z)\right)^{\prime}-\overline{z\left(H_{q}^{p}\left[\alpha_{1}\right] g(z)\right)^{\prime}}}{(1-\lambda)\left(H_{q}^{p}\left[\alpha_{1}\right] h(z)+\overline{H_{q}^{p}\left[\alpha_{1}\right] g(z)}\right)+\lambda\left(z\left(H_{q}^{p}\left[\alpha_{1}\right] h(z)\right)^{\prime}-\overline{z\left(H_{q}^{p}\left[\alpha_{1}\right] g(z)\right)^{\prime}}\right)}\right\} \\
= & \operatorname{Re}\left\{\frac{A(z)}{B(z)}\right\} \geq \gamma
\end{aligned}
$$

where

$$
A(z)=z\left(H_{q}^{p}\left[\alpha_{1}\right] h(z)\right)^{\prime}-\overline{z\left(H_{q}^{p}\left[\alpha_{1}\right] g(z)\right)^{\prime}}=z+\sum_{n=2}^{\infty} n \Gamma\left(\alpha_{1}, n\right) a_{n} z^{n}-\sum_{n=1}^{\infty} n \Gamma\left(\alpha_{1}, n\right) \bar{b}_{n} \bar{z}^{n}
$$

and $B(z)=(1-\lambda)\left(H_{q}^{p}\left[\alpha_{1}\right] h(z)+\overline{H_{q}^{p}\left[\alpha_{1}\right] g(z)}\right)+\lambda\left(z\left(H_{q}^{p}\left[\alpha_{1}\right] h(z)\right)^{\prime}-\overline{z\left(H_{q}^{p}\left[\alpha_{1}\right] g(z)\right)^{\prime}}\right)$

$$
=z+\sum_{n=2}^{\infty}(1-\lambda+n \lambda) \Gamma\left(\alpha_{1}, n\right) a_{n} z^{n}+\sum_{n=1}^{\infty}(1-\lambda-n \lambda) \Gamma\left(\alpha_{1}, n\right) \bar{b}_{n} \bar{z}^{n} .
$$

Using the fact that $\operatorname{Re}\{w\} \geq \gamma$ if and only if $|1-\gamma+w| \geq|1+\gamma-w|$, it suffices to show that

$$
|A(z)+(1-\gamma) B(z)|-|A(z)-(1+\gamma) B(z)| \geq 0 .
$$

Substituting for $A(z)$ and $B(z)$ in (2.2), we get

$$
\begin{aligned}
& |A(z)+(1-\gamma) B(z)|-|A(z)-(1+\gamma) B(z)| \\
= & \mid(2-\gamma) z+\sum_{n=2}^{\infty}[(n+1-\gamma)(1-\lambda+n \lambda)] \Gamma\left(\alpha_{1}, n\right) a_{n} z^{n}- \\
& -\sum_{n=1}^{\infty}[n-(1-\gamma)(1-\lambda+n \lambda)] \Gamma\left(\alpha_{1}, n\right) \bar{b}_{n} \bar{z}^{n} \mid
\end{aligned}
$$




$$
\begin{aligned}
& -\mid-\gamma z+\sum_{n=2}^{\infty}\left[n-(1+\gamma)(1-\lambda+n \lambda) \Gamma\left(\alpha_{1}, n\right) a_{n} z^{n}-\right. \\
& -\sum_{n=1}^{\infty}[n+(1+\gamma)(1-\lambda+n \lambda)] \Gamma\left(\alpha_{1}, n\right) \bar{b}_{n} \bar{z}^{n} \mid \\
\geq & (2-\gamma)|z|-\sum_{n=2}^{\infty}\left[n+(1-\gamma)(1-\lambda+n \lambda) \Gamma\left(\alpha_{1}, n\right)\left|a_{n}\right||z|^{n}-\right. \\
& -\sum_{n=1}^{\infty}[n-(1-\gamma)(1-\lambda-n \lambda)] \Gamma\left(\alpha_{1}, n\right)\left|b_{n}\right||z|^{n}- \\
& -\gamma|z|-\sum_{n=2}^{\infty}[n-(1+\gamma)(1-\lambda+n \lambda)] \Gamma\left(\alpha_{1}, n\right)\left|a_{n}\right||z|^{n}- \\
& -\sum_{n=1}^{\infty}[n+(1+\gamma)(1-\lambda-n \lambda)] \Gamma\left(\alpha_{1}, n\right)\left|b_{n}\right||z|^{n} \\
\geq & 2(1-\gamma)|z|\left\{2-\sum_{n=1}^{\infty}\left[\frac{n-\gamma-\gamma \lambda(n-1))}{1-\gamma}\left|a_{n}\right|+\frac{n+\gamma-\gamma \lambda(n+1))}{1-\gamma}\left|b_{n}\right|\right] \Gamma\left(\alpha_{1}, n\right)|z|^{n-1}\right\} \\
\geq & 2(1-\gamma)\left\{2-\sum_{n=1}^{\infty}\left[\frac{n-\gamma-\gamma \lambda(n-1))}{1-\gamma}\left|a_{n}\right|+\frac{n+\gamma-\gamma \lambda(n+1))}{1-\gamma}\left|b_{n}\right|\right] \Gamma\left(\alpha_{1}, n\right)\right\} .
\end{aligned}
$$

The above expression is non negative by (2.1), and so $f(z) \in \mathscr{R}_{\mathscr{H}}^{p, q}\left(\left[\alpha_{1}\right], \lambda, \gamma\right)$.

The harmonic function

$$
f(z)=z+\sum_{n=2}^{\infty} \frac{1-\gamma}{\left.(n-\gamma-\gamma \lambda(n-1)) \Gamma\left(\alpha_{1}, n\right)\right)} x_{n} z^{n}+\sum_{n=1}^{\infty} \frac{1-\gamma}{(n+\gamma-\gamma \lambda(n+1)) \Gamma\left(\alpha_{1}, n\right)} \bar{y}_{n}(\bar{z})^{n}
$$

where $\sum_{n=2}^{\infty}\left|x_{n}\right|+\sum_{n=1}^{\infty}\left|y_{n}\right|=1$ shows that the coefficient bound given by (2.1) is sharp.

The functions of the form (2.3) are in $\mathscr{R}_{\mathscr{H}}^{p, q}\left(\left[\alpha_{1}\right], \lambda, \gamma\right)$ because

$$
\begin{aligned}
& \sum_{n=1}^{\infty}\left(\frac{(n-\gamma-\gamma \lambda(n-1)) \Gamma\left(\alpha_{1}, n\right)}{1-\gamma}\left|a_{n}\right|+\frac{(n+\gamma-\gamma \lambda(n+1)) \Gamma\left(\alpha_{1}, n\right)}{1-\gamma}\left|b_{n}\right|\right) \\
= & 1+\sum_{n=2}^{\infty}\left|x_{n}\right|+\sum_{n=1}^{\infty}\left|y_{n}\right|=2 .
\end{aligned}
$$

Next theorem establishes that such coefficient bounds cannot be improved further.

Theorem 2.2. For $a_{1}=1$ and $0 \leq \gamma<1, f=h+\bar{g} \in \mathscr{R} \frac{p, q}{\mathscr{H}}\left(\left[\alpha_{1}\right], \lambda, \gamma\right)$ if and only if

$$
\sum_{n=1}^{\infty}\left[(n-\gamma-\gamma \lambda(n-1))\left|a_{n}\right|+(n+\gamma-\gamma \lambda(n+1))\left|b_{n}\right|\right] \Gamma\left(\alpha_{1}, n\right) \leq 2(1-\gamma) .
$$

Proof. Since $\mathscr{R} \frac{p, q}{\not \mathscr{C}}\left(\left[\alpha_{1}\right], \lambda, \gamma\right) \subset \mathscr{R}_{\mathscr{H}}^{p, q}\left(\left[\alpha_{1}\right], \lambda, \gamma\right)$, we only need to prove the "only if" part of the theorem. To this end, for functions $f$ of the form (1.3), we notice that the condition

$$
\operatorname{Re}\left\{\frac{z\left(H_{q}^{p}\left[\alpha_{1}\right] f(z)\right)^{\prime}}{(1-\lambda) H_{q}^{p}\left[\alpha_{1}\right] f(z)+\lambda z\left(H_{q}^{p}\left[\alpha_{1}\right] f(z)\right)^{\prime}}\right\} \geq \gamma .
$$


Equivalently,

$\operatorname{Re}\left\{\frac{(1-\gamma) z-\sum_{n=2}^{\infty}(n-\gamma-\gamma \lambda(n-1)) \Gamma\left(\alpha_{1}, n\right) a_{n} z^{n}-\sum_{n=1}^{\infty}(n+\gamma-\gamma \lambda(n+1)) \Gamma\left(\alpha_{1}, n\right) \bar{b}_{n} \bar{z}^{n}}{z-\sum_{n=2}^{\infty}(1-\lambda+n \lambda) \Gamma\left(\alpha_{1}, n\right) a_{n} z^{n}+\sum_{n=1}^{\infty}(1-\lambda-n \lambda) \Gamma\left(\alpha_{1}, n\right) \bar{b}_{n} \bar{z}^{n}}\right\} \geq 0$.

The above required condition must hold for all values of $z$ in $U$. Upon choosing the values of $z$ on the positive real axis where $0 \leq z=r<1$, we must have

$$
\frac{(1-\gamma)-\sum_{n=2}^{\infty}(n-\gamma-\gamma \lambda(n-1)) \Gamma\left(\alpha_{1}, n\right) a_{n} r^{n-1}-\sum_{n=1}^{\infty}(n+\gamma-\gamma \lambda(n+1)) \Gamma\left(\alpha_{1}, n\right) b_{n} r^{n-1}}{1-\sum_{n=2}^{\infty}(1-\lambda+n \lambda) \Gamma\left(\alpha_{1}, n\right) a_{n} r^{n-1}+\sum_{n=1}^{\infty}(1-\lambda-n \lambda) \Gamma\left(\alpha_{1}, n\right) b_{n} r^{n-1}} \geq 0 .
$$

If the condition (2.4) does not hold, then the numerator in (2.5) is negative for $r$ sufficiently close to 1 . Hence, there exist $z_{0}=r_{0}$ in $(0,1)$ for which the quotient of (2.5) is negative. This contradicts the required condition for $f(z) \in \mathscr{R} \frac{p, q}{\not \mathscr{C}}\left(\left[\alpha_{1}\right], \lambda, \gamma\right)$. This completes the proof of the theorem.

Letting $p=1$ and $q=\lambda=0$ in Theorem 2.2, we have

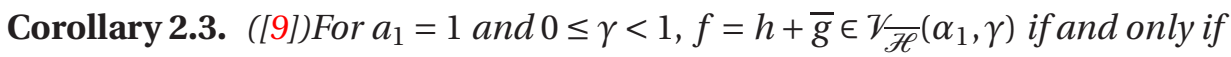

$$
\sum_{n=1}^{\infty}\left[(n-\gamma)\left|a_{n}\right|+(n+\gamma(n+1))\left|b_{n}\right|\right] \Gamma\left(\alpha_{1}, n\right) \leq 2(1-\gamma) .
$$

Letting $p=1, q=0$ and $\alpha_{1}=1$ in Theorem 2.2, we have

Corollary 2.4. ([10])For $a_{1}=1$ and $0 \leq \gamma<1, f=h+\bar{g} \in \mathscr{T}_{\mathscr{S}_{\mathscr{H}}^{*}}(\lambda, \gamma)$ if and only if

$$
\sum_{n=1}^{\infty}\left[(n-\gamma-\gamma \lambda(n-1))\left|a_{n}\right|+(n+\gamma-\gamma \lambda(n+1))\left|b_{n}\right|\right] \leq 2(1-\gamma) .
$$

Letting $p=1, q=0$ and $\alpha_{1}=2$ in Theorem 2.2, we have

Corollary 2.5. For $a_{1}=1$ and $0 \leq \gamma<1, f=h+\bar{g} \in \mathscr{R} \frac{1,0}{\mathscr{H}}([2], \lambda, \gamma)$ if and only if

$$
\sum_{n=1}^{\infty} n\left[(n-\gamma-\gamma \lambda(n-1))\left|a_{n}\right|+(n+\gamma-\gamma \lambda(n+1))\left|b_{n}\right|\right] \leq 2(1-\gamma) .
$$

Letting $p=1, q=0$ and $\alpha_{1}=3$ in Theorem 2.2, we have

Corollary 2.6. For $a_{1}=1$ and $0 \leq \gamma<1, f=h+\bar{g} \in \mathscr{R} \frac{1,0}{\mathscr{H}}([3], \lambda, \gamma)$ if and only if

$$
\sum_{n=1}^{\infty} \frac{n(n+1)}{2}\left[(n-\gamma-\gamma \lambda(n-1))\left|a_{n}\right|+(n+\gamma-\gamma \lambda(n+1))\left|b_{n}\right|\right] \leq 2(1-\gamma) .
$$




\section{Distortion bounds and extreme points}

The following theorem gives the distortion bounds for functions in $\mathscr{R} \frac{p, q}{\mathscr{H}}\left(\left[\alpha_{1}\right], \lambda, \gamma\right)$ which yields a covering result for the class $\mathscr{R} \frac{p, q}{\mathscr{H}}\left(\left[\alpha_{1}\right], \lambda, \gamma\right)$.

Theorem 3.1. Let $f \in \mathscr{R} \frac{p, q}{\mathscr{H}}\left(\left[\alpha_{1}\right], \lambda, \gamma\right)$. Then for $|z|=r<1$, we have

$$
\begin{aligned}
& \left(1-b_{1}\right) r-\frac{\beta_{1}}{\alpha_{1}}\left(\frac{1-\gamma}{2-\gamma-\gamma \lambda}-\frac{1+\gamma}{2-\gamma-\gamma \lambda} b_{1}\right) r^{2} \leq|f(z)| \\
\leq & \left(1+b_{1}\right) r+\frac{\beta_{1}}{\alpha_{1}}\left(\frac{1-\gamma}{2-\gamma-\gamma \lambda}-\frac{1+\gamma}{2-\gamma-\gamma \lambda} b_{1}\right) r^{2} .
\end{aligned}
$$

Proof. We only prove the right hand inequality. Taking the absolute value of $f(z)$, we obtain

$$
\begin{aligned}
|f(z)| & =\left|z+\sum_{n=2}^{\infty} a_{n} z^{n}+\sum_{n=1}^{\infty} \bar{b}_{n} \bar{z}^{n}\right| \\
& \leq\left(1+b_{1}\right)|z|+\sum_{n=2}^{\infty}\left(a_{n}+b_{n}\right)|z|^{n} \\
& \leq\left(1+b_{1}\right) r+\sum_{n=2}^{\infty}\left(a_{n}+b_{n}\right) r^{2} \\
& \leq\left(1+b_{1}\right) r+\frac{(1-\gamma) \beta_{1}}{(2-\gamma-\gamma \lambda) \alpha_{1}} \sum_{n=2}^{\infty}\left(\frac{(2-\gamma-\gamma \lambda) \alpha_{1}}{(1-\gamma) \beta_{1}} a_{n}+\frac{(2-\gamma-\gamma \lambda)) \alpha_{1}}{(1-\gamma) \beta_{1}} b_{n}\right) r^{2} \\
& \leq\left(1+b_{1}\right) r+\frac{(1-\gamma) \beta_{1}}{(2-\gamma-\gamma \lambda) \alpha_{1}}\left(1-\frac{1+\gamma}{1-\gamma} b_{1}\right) r^{2} \\
& \leq\left(1+b_{1}\right) r+\frac{\beta_{1}}{\alpha_{1}}\left(\frac{1-\gamma}{2-\gamma-\gamma \lambda}-\frac{1+\gamma}{2-\gamma-\gamma \lambda} b_{1}\right) r^{2} .
\end{aligned}
$$

The proof of the left hand inequality follows on lines similar to that of the right hand side inequality.

The covering result follows from the left hand inequality given in Theorem 3.1.

Corollary 3.2. If $f(z) \in \mathscr{R} \frac{p, q}{\mathscr{H}}\left(\left[\alpha_{1}\right], \lambda, \gamma\right)$. Then

$$
\left\{w:|w|<\frac{2 \alpha_{1}-\beta_{1}-\left((1+\lambda) \alpha_{1}-\beta_{1}\right) \gamma}{(2-\gamma-\gamma \lambda) \alpha_{1}}\left(1-b_{1}\right)\right\} \subset f(U) .
$$

Proof. Using the left hand inequality of Theorem 3.1 and letting $r \rightarrow 1$, we prove that

$$
\begin{aligned}
& \left(1-b_{1}\right)-\frac{1}{\Gamma\left(\alpha_{1}, 2\right)}\left(\frac{1-\gamma}{2-\gamma-\gamma \lambda}-\frac{1+\gamma}{2-\gamma-\gamma \lambda} b_{1}\right) \\
& =\left(1-b_{1}\right)-\frac{1}{\Gamma\left(\alpha_{1}, 2\right)(2-\gamma-\gamma \lambda)}\left[1-\gamma-(1+\gamma) b_{1}\right] \\
& =\frac{\left(1-b_{1}\right) \Gamma\left(\alpha_{1}, 2\right)(2-\gamma-\gamma \lambda)-(1-\gamma)+(1+\gamma) b_{1}}{\Gamma\left(\alpha_{1}, 2\right)(2-\gamma-\gamma \lambda)}
\end{aligned}
$$




$$
=\left\{\frac{2 \alpha_{1}-\beta_{1}-\left((1+\lambda) \alpha_{1}-\beta_{1}\right) \gamma}{(2-\gamma-\gamma \lambda) \alpha_{1}}\left(1-b_{1}\right)\right\} \subset f(U)
$$

Next we determine the extreme points of closed convex hulls of $\mathscr{R} \frac{p, q}{\mathscr{P}}\left(\left[\alpha_{1}\right], \lambda, \gamma\right)$ denoted by $\operatorname{clco} \mathscr{R} \frac{p, q}{\not \mathscr{\ell}}\left(\left[\alpha_{1}\right], \lambda, \gamma\right)$.

Theorem 3.3. A function $f(z) \in \mathscr{R} \frac{p, q}{\mathscr{H}}\left(\left[\alpha_{1}\right], \lambda, \gamma\right)$ if and only if $f(z)=\sum_{n=1}^{\infty}\left(X_{n} h_{n}(z)+Y_{n} g_{n}(z)\right)$ where

$$
\begin{gathered}
h_{1}(z)=z, h_{n}(z)=z-\frac{1-\gamma}{(n-\gamma-\gamma \lambda(n-1)) \Gamma\left(\alpha_{1}, n\right)} z^{n} ; \quad(n \geq 2), \\
g_{n}(z)=z+\frac{1-\gamma}{(n+\gamma-\gamma \lambda(n+1)) \Gamma\left(\alpha_{1}, n\right)} \bar{z}^{n} ;(n \geq 2), \\
\sum_{n=1}^{\infty}\left(X_{n}+Y_{n}\right)=1, X_{n} \geq 0 \text { and } Y_{n} \geq 0 .
\end{gathered}
$$

In particular, the extreme points of $\mathscr{R} \frac{p, q}{\mathscr{H}}\left(\left[\alpha_{1}\right], \lambda, \gamma\right)$ are $\left\{h_{n}\right\}$ and $\left\{g_{n}\right\}$.

Proof. First, we note that for $f$ as in the theorem above, we may write

$$
\begin{aligned}
f(z)= & \sum_{n=1}^{\infty}\left(X_{n} h_{n}(z)+Y_{n} g_{n}(z)\right) \\
= & \sum_{n=1}^{\infty}\left(X_{n}+Y_{n}\right) z-\sum_{n=2}^{\infty} \frac{1-\gamma}{(n-\gamma-\gamma \lambda(n-1)) \Gamma\left(\alpha_{1}, n\right)} X_{n} z^{n} \\
& +\sum_{n=1}^{\infty} \frac{1-\gamma}{(n+\gamma-\gamma \lambda(n+1)) \Gamma\left(\alpha_{1}, n\right)} Y_{n} \bar{z}^{n} \\
= & z-\sum_{n=2}^{\infty} A_{n} z^{n}+\sum_{n=1}^{\infty} B_{n} \bar{z}^{n}
\end{aligned}
$$

where $\quad A_{n}=\frac{1-\gamma}{(n-\gamma-\gamma \lambda(n-1))) \Gamma\left(\alpha_{1}, n\right)} X_{n}$, and $B_{n}=\frac{1-\gamma}{(n+\gamma-\gamma \lambda(n+1)) \Gamma\left(\alpha_{1}, n\right)} Y_{n}$.

Therefore

$$
\begin{aligned}
& \sum_{n=2}^{\infty} \frac{(n-\gamma-\gamma \lambda(n-1)) \Gamma\left(\alpha_{1}, n\right)}{1-\gamma} A_{n}+\sum_{n=1}^{\infty} \frac{(n+\gamma-\gamma \lambda(n+1)) \Gamma\left(\alpha_{1}, n\right)}{1-\gamma} B_{n} \\
= & \sum_{n=2}^{\infty} X_{n}+\sum_{n=1}^{\infty} Y_{n} \\
= & 1-X_{1} \leq 1,
\end{aligned}
$$

and hence $f(z) \in \operatorname{clco} \mathscr{R} \frac{p, q}{\mathscr{H}}\left(\left[\alpha_{1}\right], \lambda, \gamma\right)$. Conversely, suppose that $f(z) \in \operatorname{clco} \mathscr{R} \frac{p, q}{\mathscr{H}}\left(\left[\alpha_{1}\right], \lambda, \gamma\right)$. Setting

$$
X_{n}=\frac{(n-\gamma-\gamma \lambda(n-1)) \Gamma\left(\alpha_{1}, n\right)}{1-\gamma} A_{n}, \quad(n \geq 2) \text { and } Y_{n}=\frac{(n+\gamma-\gamma \lambda(n-1)) \Gamma\left(\alpha_{1}, n\right)}{1-\gamma} B_{n}, \quad(n \geq 1)
$$


where $\sum_{n=1}^{\infty}\left(X_{n}+Y_{n}\right)=1$. Then

$$
\begin{aligned}
f(z) & =z-\sum_{n=2}^{\infty} a_{n} z^{n}+\sum_{n=1}^{\infty} \bar{b}_{n} \bar{z}^{n}, a_{n}, b_{n} \geq 0 . \\
& =z-\sum_{n=2}^{\infty} \frac{1-\gamma}{(n-\gamma-\gamma \lambda(n-1)) \Gamma\left(\alpha_{1}, n\right)} X_{n} z^{n}+\sum_{n=1}^{\infty} \frac{1-\gamma}{(n+\gamma-\gamma \lambda(n-1)) \Gamma\left(\alpha_{1}, n\right)} Y_{n} \bar{z}^{n} \\
& =z-\sum_{n=2}^{\infty}\left(h_{n}(z)-z\right) X_{n}+\sum_{n=1}^{\infty}\left(g_{n}(z)-z\right) Y_{n} \\
& =\sum_{n=1}^{\infty}\left(X_{n} h_{n}(z)+Y_{n} g_{n}(z)\right)
\end{aligned}
$$

as required.

\section{Inclusion results}

Now we show that $\mathscr{R} \frac{p, q}{\not \mathscr{C}}\left(\left[\alpha_{1}\right], \lambda, \gamma\right)$ is closed under convex combinations of its member and also closed under the convolution product.

Theorem 4.1. The family $\mathscr{R} \frac{p, q}{\mathscr{H}}\left(\left[\alpha_{1}\right], \lambda, \gamma\right)$ is closed under convex combinations.

Proof. For $i=1,2, \ldots$, suppose that $f_{i} \in \mathscr{R} \frac{p, q}{\mathscr{H}}\left(\left[\alpha_{1}\right], \lambda, \gamma\right)$ where

$$
f_{i}(z)=z-\sum_{n=2}^{\infty} a_{i, n} z^{n}+\sum_{n=2}^{\infty} \bar{b}_{i, n} \bar{z}^{n}
$$

Then, by Theorem 3.1

$$
\sum_{n=2}^{\infty} \frac{(n-\gamma-\gamma \lambda(n-1)) \Gamma\left(\alpha_{1}, n\right)}{(1-\gamma)} a_{i, n}+\sum_{n=1}^{\infty} \frac{(n+\gamma-\gamma \lambda(n+1)) \Gamma\left(\alpha_{1}, n\right)}{(1-\gamma)} b_{i, n} \leq 1 .
$$

For $\sum_{i=1}^{\infty} t_{i}, 0 \leq t_{i} \leq 1$, the convex combination of $f_{i}$ may be written as

$$
\sum_{i=1}^{\infty} t_{i} f_{i}(z)=z-\sum_{n=2}^{\infty}\left(\sum_{i=1}^{\infty} t_{i} a_{i, n}\right) z^{n}+\sum_{n=1}^{\infty}\left(\sum_{i=1}^{\infty} t_{i} \bar{b}_{i, n}\right) \bar{z}^{n} .
$$

Using the inequality (2.4), we obtain

$$
\begin{aligned}
& \sum_{n=2}^{\infty} \frac{(n-\gamma-\gamma \lambda(n-1)) \Gamma\left(\alpha_{1}, n\right)}{(1-\gamma)}\left(\sum_{i=1}^{\infty} t_{i} a_{i, n}\right)+\sum_{n=1}^{\infty} \frac{(n+\gamma-\gamma \lambda(n+1)) \Gamma\left(\alpha_{1}, n\right)}{(1-\gamma)}\left(\sum_{i=1}^{\infty} t_{i} b_{i, n}\right) \\
= & \sum_{i=1}^{\infty} t_{i}\left(\sum_{n=2}^{\infty} \frac{(n-\gamma-\gamma \lambda(n-1)) \Gamma\left(\alpha_{1}, n\right)}{(1-\gamma)} a_{i, n}+\sum_{n=1}^{\infty} \frac{(n+\gamma-\gamma \lambda(n+1)) \Gamma\left(\alpha_{1}, n\right)}{(1-\gamma)} b_{i, n}\right) \\
\leq & \sum_{i=1}^{\infty} t_{i}=1,
\end{aligned}
$$

and therefore $\sum_{i=1}^{\infty} t_{i} f_{i} \in \mathscr{R} \frac{p, q}{\mathscr{H}}\left(\left[\alpha_{1}\right], \lambda, \gamma\right)$. 
Theorem 4.2. For $0 \leq \beta \leq \gamma<1$, let $f(z) \in \mathscr{R} \frac{p, q}{\mathscr{H}}\left(\left[\alpha_{1}\right], \lambda, \gamma\right)$ and $F(z) \in \mathscr{R} \frac{p, q}{\mathscr{H}}\left(\left[\alpha_{1}\right], \lambda, \delta\right)$. Then $f(z) * F(z) \in \mathscr{R} \frac{p, q}{\mathscr{P}}\left(\left[\alpha_{1}\right], \lambda, \gamma\right) \subset \mathscr{R} \frac{p, q}{\not \mathscr{C}}\left(\left[\alpha_{1}\right], \lambda, \delta\right)$.

Proof. Let $f(z)=z-\sum_{n=2}^{\infty} a_{n} z^{n}+\sum_{n=1}^{\infty} \bar{b}_{n} \bar{z}^{n} \in \mathscr{R} \overline{\not \ell}\left(\left[\alpha_{1}\right], \lambda, \gamma\right)$ and $F(z)=z-\sum_{n=2}^{\infty} A_{n} z^{n}+\sum_{n=1}^{\infty} \bar{B}_{n} \bar{z}^{n} \in$ $\mathscr{R} \frac{p, q}{\mathscr{H}}\left(\left[\alpha_{1}\right], \lambda, \delta\right)$. Then $f(z) * F(z)$ is $f(z) * F(z)=z-\sum_{n=2}^{\infty} a_{n} A_{n} z^{n}+\sum_{n=1}^{\infty} \bar{b}_{n} \bar{B}_{n} \bar{z}^{n}$.

For $f(z) * F(z) \in \mathscr{R} \frac{p, q}{\mathscr{H}}\left(\left[\alpha_{1}\right], \lambda, \delta\right)$ we note that $\left|A_{n}\right| \leq 1$ and $\left|B_{n}\right| \leq 1$. Now by Theorem 2.2, we have

$$
\begin{aligned}
& \sum_{n=2}^{\infty} \frac{(n-\delta-\delta \lambda(n-1)) \Gamma\left(\alpha_{1}, n\right)}{1-\delta}\left|a_{n}\right|\left|A_{n}\right|+\sum_{n=1}^{\infty} \frac{(n+\delta-\delta \lambda(n+1)) \Gamma\left(\alpha_{1}, n\right)}{1-\delta}\left|b_{n}\right|\left|B_{n}\right| \\
& \leq \sum_{n=2}^{\infty} \frac{(n-\gamma-\gamma \lambda(n-1)) \Gamma\left(\alpha_{1}, n\right)}{1-\gamma}\left|a_{n}\right|+\sum_{n=1}^{\infty} \frac{[n+\gamma-\gamma \lambda(n+1)) \Gamma\left(\alpha_{1}, n\right)}{1-\gamma}\left|b_{n}\right| \leq 1,
\end{aligned}
$$

by Theorem 2.2, $f(z) \in \mathscr{R} \frac{p, q}{\mathscr{H}}\left(\left[\alpha_{1}\right], \lambda, \gamma\right)$. Therefore $f(z) * F(z) \in \mathscr{R} \frac{p, q}{\mathscr{H}}\left(\left[\alpha_{1}\right], \lambda, \gamma\right) \subset \mathscr{R} \frac{p, q}{\mathscr{H}}\left(\left[\alpha_{1}\right], \lambda, \delta\right)$.

\section{References}

[1] B. C. Carlson, Special Functions of Applied Mathematics, Academic Press, New York, 1977.

[2] B. C. Carlson and S. B.Shaffer, Starlike and prestarlike hypergeometric functions, SIAM, J. Math. Anal., 15 (2002), 737-745.

[3] J. Clunie and T. Sheil-Small, Harmonic univalent functions, Ann. Acad. Aci. Fenn. Ser. A. I. Math., 9 (1984), $3-25$.

[4] J. Dziok and H. M. Srivastava, Certain subclasses of analytic functions associated with the generalized hypergeometric function, Integral Transform Spec. Funct., 14 (2003), 7-18.

[5] J. M. Jahangiri and H. Silverman, Harmonic univalent functions with varying arguments, Internat. J. Appl. Math., 8(2002), 267-275.

[6] J. M. Jahangiri, Harmonic functions starlike in the unit disc, J. Math. Anal. Appl., 235(1999), 470-477.

[7] J. M. Jahangiri, G. Murugusundaramoorthy and K. Vijaya, Starlikeness of Rucheweyh type harmonic univalent functions, J. Indian Acad. Math., 26 (2004), 191-200.

[8] J. M. Jahangiri, G. Murugusundaramoorthy and K. Vijaya, Salagean-Type harmonic univalent functions, Southwest J. Pure Appl. Math., 2 (2002), 77-82.

[9] H. A. Al-Kharsani and R. A. Al-Khal, Univalent harmonic functions, J. Inequal. Pure Appl. Math., 8 (2007), Issue 2, Art. 59, 8 pp.

[10] M. Öztürk, S. Yalçin and M. Yamankaradenz, Convex subclass of harmonic starlike functions, Appl. Math. Comp., 154, 2(5) (2004), 449-459.

[11] S. Ponnusamy and S. Sabapathy, Geometric properties of generalized hypergeometric functions, Ramanujan J., 1 (1997), 187-210.

[12] E. D. Rainville, Special Functions, Chelsea Publishing Company, New York 1960.

[13] S. Ruscheweyh, New criteria for univalent functions, Proc. Amer. Math. Soc., 49 (1975), 109-115.

[14] H. Silverman, Harmonic univalent functions with negative coefficients, J. Math. Anal. Appl., 220 (1998), 283289.

[15] H. M. Srivastava and S. Owa, Some characterization and distortion theorems involving fractional calculus, generalized hypergeometric functions, Hadamard products, linear operators and certain subclasses of analytic functions, Nagoya Math. J., 106 (1987), 1-28. 
[16] K. Vijaya, Studies on certain subclasses of harmonic functions, Ph.D. Thesis, VIT University, September 2006.

${ }^{1}$ School of Advanced Sciences and Humanities, V I T University, Vellore - 632014,T.N.,India.

E-mail: gmsmoorthy@yahoo.com

${ }^{2}$ School of Advanced Sciences and Humanities, V I T University, Vellore - 632014,T.N.,India.

E-mail: kvavit@yahoo.co.in

${ }^{3}$ Department of Mathematics, Al al-Bayt University, P.O. Box: 130095 Mafraq, Jordan.

E-mail: bafrasin@yahoo.com 